\title{
Prontuário Eletrônico do Paciente baseado em blockchain: Um Desenho de Pesquisa Sociotécnico
}

\author{
Pamella Soares $^{1 凶}$, Allysson Allex Araújo ${ }^{2}$, Raphael Saraiva ${ }^{1}$, \\ Rayane Santos $^{1}$ e Jerffeson Souza ${ }^{1}$
}

\author{
${ }^{1}$ Programa de Pós-Graduação em Ciência da Computação (PPGCC) \\ Universidade Estadual do Ceará (UECE) - Fortaleza, Ceará - Brasil \\ ${ }^{2}$ Grupo de Estudos em Sistemas de Informação e Inovação Digital (GESID) \\ Universidade Federal do Ceará (UFC) - Crateús, Ceará - Brasil \\ pamella.soares@aluno. uece.br
}

\begin{abstract}
The Electronic Patient Record (EPR) has transformed how clinical information is managed. In this context, blockchain is particularly promising due to its capability to allow completely anonymous entities to form a network that stores reliable information, thus enabling the creation of new social spaces for collaborative systems. However, we identified a research gap regarding the understanding of the effect of blockchain's adoption in EPR in the light of collaborative constructs. Therefore, this paper presents a research design drawing from a socio-technical perspective for developing and evaluating a blockchainbased solution for EPR through which the patient can share the medical data with different entities in a secure way. In summary, we contribute by presenting a new methodological framework in the domain blockchain for health.
\end{abstract}

Resumo. O Prontuário Eletrônico do Paciente (PEP) transformou a forma como as informações clínicas são gerenciadas. Nesse contexto, a adoção de blockchain se demonstra particularmente promissora devido sua capacidade de permitir que partes completamente anônimas formem uma rede que armazena informações confiáveis, propiciando, assim, a criação de novos espaços sociais a partir de sistemas colaborativos. Entretanto, identifica-se na literatura uma lacuna quanto ao entendimento sobre o efeito da adoção de blockchain no contexto de PEP à luz de construtos colaborativos. Dessa forma, o presente trabalho apresenta um desenho de pesquisa pautado em uma perspectiva sociotécnica para desenvolvimento e avaliação de uma solução para PEP baseada em blockchain pela qual o paciente pode compartilhar seus dados médicos com diferentes entidades de forma segura. Contribui-se, portanto, ao apresentar um novo enquadramento metodológico no domínio de blockchain para saúde.

\section{Introdução}

A partir das limitações oriundas do uso de prontuário em papel, deu-se início ao desenvolvimento do Prontuário Eletrônico do Paciente (PEP), uma estrutura para manutenção da informação eletrônica sobre o estado de saúde do indivíduo e os cuidados recebidos durante sua vida [Massad et al. 2003]. Além das finalidades de armazenamento, comunicação e garantia da continuidade do cuidado e do gerenciamento das unidades de saúde, os dados armazenados no PEP favorecem a interoperabilidade entre diferentes 
partes, tanto em prol da colaboração entre os profissionais de saúde como fonte para estudos de extração de conhecimento em pesquisas. Nesse contexto, outro requisito crítico é a garantia da privacidade quanto aos dados dos pacientes [Ricarte 2019] alicerçada pela Lei Geral de Proteção de Dados Pessoais (LGPD). Dessa forma, uma questão pertinente é como prover acesso aberto a dados sensíveis de modo seguro e que preserve a privacidade e anonimidade dos pacientes [da Conceição et al. 2018]. Diante de tais particularidades, a tecnologia blockchain se demonstra promissora por sua viabilização de que partes anônimas e que não confiam entre si formem uma rede que armazena informações confiáveis, garantindo a auditabilidade, disponibilidade e integridade das transações armazenadas. Em termos genéricos, o blockchain é um banco de dados distribuído, protegido por criptografia e governado por um mecanismo de consenso [Beck et al. 2017], ou seja, um registro de eventos transparente, seguro e resiliente.

Com o apoio de blockchain, as pessoas, as instituições e os diferentes setores, colaboram entre si para a solução de diferentes problemas, sendo possível a criação dos atuais espaços sociais para o trabalho por meio de sistemas colaborativos. Especificamente, a área de Trabalho Cooperativo Suportado por Computador (inglês Computer Supported Cooperative Work ou CSCW) conceitua a construção de sistemas que atendem tanto o ponto de vista da tecnologia, quanto aos efeitos psicológicos, sociais e organizacionais do trabalho em grupo [Nicolaci-da Costa and Pimentel 2011]. Nesse sentido, sabe-se que a adoção de novas tecnologias, como blockchain, pode transformar, de forma significativa, as várias dimensões de um contexto real da sociedade [Prinz 2018]. Conforme [Cukierman et al. 2007], tais mudanças influenciam nas relações e comunicações interpessoais, desde negócios até questões políticas. Apesar disso, a adoção de blockchain no contexto de PEP tem sido avaliada apenas sob o ponto de vista técnico, denotando, assim, uma escassez de estudos que buscam compreender os relevantes efeitos da tecnologia sobre as pessoas, de forma individual e coletiva.

Para mitigar tais lacunas, a abordagem sociotécnica se demonstra relevante, pois, além de se preocupar com o viés tecnológico, considera tanto o contexto de trabalho no processo de desenvolvimento, quanto à análise de como usuários são impactados ao interagirem com a tecnologia [Bassan de Moraes et al. 2019]. Dessa forma, ao fundamentarse numa perspectiva sociotécnica, torna-se possível, por exemplo, concretizar análises sobre o atendimento dos pilares de comunicação, coordenação, cooperação e colaboração entre os usuários [Costa et al. 2014], elementos estes especialmente relevantes no contexto do compartilhamento das informações médicas através do PEP.

Considerando tal motivação, este trabalho apresenta um desenho metodológico multi-método, baseado em um escopo sociotécnico e alicerçado por Design Science Research (DSR) [Vaishnavi and Kuechler 2004], para nortear o desenvolvimento e a avaliação de uma solução baseada em blockchain que possibilita o paciente compartilhar seus dados médicos. Este trabalho contribui ao apresentar um enquadramento metodológico sociotécnico no domínio de blockchain para saúde alinhado às normas técnicas estabelecidas pela Sociedade Brasileira de Informática em Saúde (SBIS) e LGPD.

\section{Trabalhos Relacionados}

Agostinho et al. (2019) apresentam uma solução baseada em blockchain para o problema de interoperabilidade de dados médicos. A abordagem utiliza assinatura assimétrica para 
garantir a confidencialidade dos dados entre os participantes da rede. Por sua vez, Viana et al. (2020) sugerem um sistema de gerência de prontuário por pacientes em processo de reabilitação física e neuro-funcional, possibilitando a configuração do acesso à outras entidades de saúde. Zhang et al. (2018) apresentam uma arquitetura para compartilhamento de dados clínicos baseada em blockchain e no padrão HL7 Fast Healthcare Interoperability Resources, e aderente aos requisitos do National Coordinator for Health Information Technology. McFarlane et al. (2017) propõem o Patientory, um sistema que permite a interação paciente-provedor através da blockchain, visando reduzir custos e baseando-se nos padrões da Health Insurance Portability and Accountability Act.

Os trabalhos mencionados apresentam abordagens para confiabilidade, privacidade e controle de acesso. Dentre eles, alguns realizam adequações conforme regimentos internacionais estabelecidos para proteção de dados. Porém, observa-se que apresentam lacunas quanto à execução de uma avaliação sociotécnica. Especificamente, nenhum dos trabalhos observados teve como objetivo investigar o impacto das soluções em questões relacionadas à comunicação, coordenação, cooperação e colaboração entre os usuários. Adicionalmente, identificou-se que as propostas nacionais não apresentaram uma análise quanto a sua aderência aos requisitos técnicos estabelecidos pela SBIS e LGPD.

\section{Procedimentos Metodológicos}

Nesta seção, serão apresentadas a natureza da presente pesquisa e os procedimentos metodológicos a serem utilizados em cada etapa.

\subsection{Caracterização da Pesquisa}

A natureza metodológica desta proposta é classificada como uma abordagem quali-quantitativa do tipo exploratória alicerçada em Design Science Research (DSR) [Vaishnavi and Kuechler 2004], como apresentado na Figura 1. A primeira fase inicia-se com a Conscientização do Problema a qual se planeja conduzir uma revisão de literatura e análise documental. Assim, torna-se possível identificar e analisar estudos relacionadas ao desenvolvimento de abordagens referentes à presente pesquisa, além do fornecimento de suporte teórico. Quanto à análise documental [Bowen et al. 2009], tem-se o entendimento do arcabouço regimental para adequação da proposta ao cenário brasileiro em termos do desenvolvimento de PEP e alinhamento à LGPD. Em relação à fase de Sugestão, propõe-se, como tentative design [Beck et al. 2013], a definição de um modelo arquitetural que represente uma visão abstrata do artefato a ser construído sob a forma de uma aplicação web. Em seguida, na fase de Desenvolvimento, cujo foco consiste na implementação do artefato propriamente dito, planeja-se a construção de i) um contrato inteligente com a regra de negócio a ser executada na rede; (ii) uma Application Programming Interface para comunicação com a blockchain; (iii) a integração com sistemas de armazenamento distribuído; e (iv) uma interface a partir de uma aplicação web. Na Fase de Avaliação, tem-se a condução de uma análise empírica com foco em questões sociotécnicas como Utilidade Percebida [Davis 1989], fatores colaborativos [Costa et al. 2014], aderência de requisitos e desempenho computacional. Finalmente, na etapa de Conclusão, os resultados do esforço de pesquisa são consolidados e tem-se a discussão do conhecimento adquirido. 


\begin{tabular}{|c|c|c|c|c|}
\hline $\begin{array}{c}\text { Conscientização do } \\
\text { Problema }\end{array}$ & Sugestão & Desenvolvimento & Avaliação & Conclusão \\
\hline $\begin{array}{l}\text { Processo: identificar e } \\
\text { compreender o problema } \\
\text { que deseja estudar }\end{array}$ & $\begin{array}{l}\text { Processo: criação de um } \\
\text { Tentative Design (ou design } \\
\text { provisório) do artefato }\end{array}$ & $\begin{array}{l}\text { Processo: implementação do } \\
\text { artefato proposto }\end{array}$ & $\begin{array}{l}\text { Processo: avaliação experimental } \\
\text { social e técnica }\end{array}$ & \multirow{2}{*}{$\begin{array}{l}\text { Processo: discussão do conhe- } \\
\text { cimento adquirido a partir } \\
\text { das análises e avaliações } \\
\text { realizadas }\end{array}$} \\
\hline $\begin{array}{l}\text { Método: } \\
\text { - Revisão bibliográfica } \\
\text { - Análise documental em } \\
\text { normas técnicas (SBIS e } \\
\text { LGPD) }\end{array}$ & $\begin{array}{l}\text { Método: } \\
\text { - Definição dos requisitos } \\
\text { advindos das normas técnicas } \\
\text { - Projeto do modelo arquite- } \\
\text { tural proposto }\end{array}$ & $\begin{array}{l}\text { Método: } \\
\text { - Implementação do contrato } \\
\text { inteligente, API de comunica- } \\
\text { ção, integração com sistema } \\
\text { distribuído e front-end }\end{array}$ & $\begin{array}{l}\text { Método: } \\
\text { - Avaliação Social (Observação } \\
\text { Participante e Grupo Focal); } \\
\text { - Avaliação de Técnica (Análise } \\
\text { de Aderência dos Requisitos e de } \\
\text { Desempenho) }\end{array}$ & \\
\hline
\end{tabular}

Figura 1. Procedimentos Metodológicos baseados em DSR.

\subsection{Design da Avaliação}

Em relação à Avaliação Social, planeja-se um quasi-experimento baseado na realização de tarefas com um grupo de usuários os quais deverão interagir sequencialmente com a interface do artefato [Nielsen 1994]. A realização de experimentos em grupo se demonstra relevante para o presente estudo haja visto que possibilita uma visão complementar e compartilhada do uso da solução. Conforme ilustrado na Figura 2, este processo é guiado através de um roteiro baseado em cinco etapas: 1) explicação dos procedimentos, 2) preenchimento do Termo de Consentimento Livre e Esclarecido, 3) o preenchimento de um formulário para a caracterização dos participantes, 4) a Observação Participante da realização de tarefas derivadas a partir de um workflow de modo que os usuários usufruam da técnica do Think-Aloud [Rubin and Chisnell 2008] e, por fim, 5) condução de um Grupo Focal orientando-se a partir de três perspectivas alicerçadas na teoria sociotécnica: (i) a Utilidade Percebida proveniente do Technology Acceptance Model [Davis 1989], ou seja, quanto uma pessoa acredita que o uso de um determinado sistema melhora seu desempenho no trabalho, (ii) o contexto de aplicação, isto é, o uso de PEP baseado em blockchain; e (iii) os fatores de comunicação, coordenação, colaboração e cooperação delineados no Modelo 4C [Costa et al. 2014] à luz das particularidades do artefato proposto. Em específico, o usufruto do Grupo Focal se demonstra útil visto que pode-se coletar, por meio de interação do grupo, insights sobre as questões investigadas [Morgan 1996]. O guia para condução da Observação Participante contendo cenários e suas respectivas tarefas, e do Grupo Focal, incluindo as perguntas a serem realizadas, encontram-se disponíveis na página de suporte ${ }^{1}$.

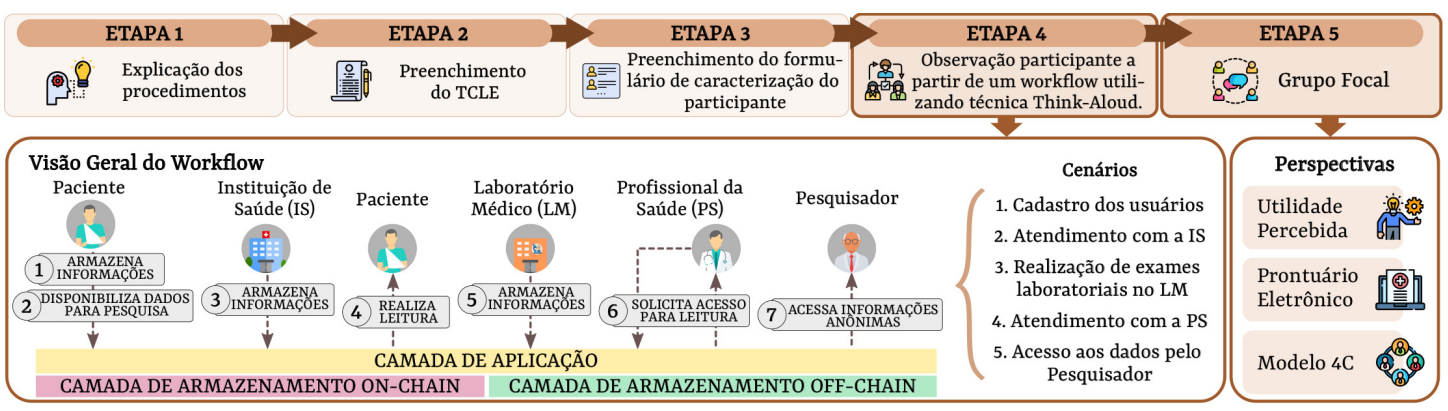

Figura 2. Fluxo da Avaliação Social.

Em específico, na Etapa 4, estabeleceu-se uma visão geral do workflow hipotético para derivação e execução das cenários específicos em conformidade às normas vigentes [CFM 2002]. Identificam-se, portanto, cinco perfis de usuário, são eles: i) Paciente -

${ }^{1}$ https://pamellasds.github.io/pep-blockchain 
indivíduo cuidado por um profissional da área da saúde; ii) Profissional da Saúde (PS) profissional cuja área de atuação está relacionada às ciências da saúde; iii) Instituição de Saúde (IS) - estabelecimento que assegura assistência médica; iv) Laboratório Médico (LM) - local onde testes patológicos são realizados e; v) Pesquisador - o profissional que utilizará os dados anonimizados para pesquisa. No Passo 1, ao acessar o sistema, o Paciente poderá cadastrar seus dados pessoais através da Camada de Aplicação e, no Passo 2, pode decidir se seus registros médicos podem ser armazenados, de forma anônima, em um banco de dados de pesquisa. Já no Passo 3, por sua vez, o paciente necessita ir a uma IS a qual solicita todas as informações necessárias para preencher uma Anamnese referente ao paciente, por exemplo. No Passo 4, o Paciente realiza a leitura de seu histórico ou informações clínicas registradas no sistema. No Passo 5, supondo que a IS tenha solicitado um exame laboratorial, o LM armazena os resultados dos exames requisitados, referenciando o paciente como sendo o detentor de tal Registro. Isso possibilitará que apenas o Paciente tenha controle sobre suas informações médicas, uma vez gravado em blockchain. Ao submeter-se a uma consulta, por exemplo, o paciente pode permitir que um determinado médico seja associado a um Registro específico (Passo 6), tal controle de acesso é gravado em blockchain e habilitará o médico a visualizar informações necessárias. Por fim, no Passo 7, visto que o paciente autorizou seus dados anônimos para a pesquisa, o pesquisador poderá coletar as informações anônimas dos registros armazenados na rede. Ressalta-se que as tarefas dos cenários são definidas com o objetivo de analisar os aspectos colaborativos na utilização de PEP baseado em blockchain.

Por sua vez, na Avaliação Técnica tem-se, inicialmente, a análise de aderência dos requisitos advindos de Normas Técnicas (SBIS e LGPD) com o objetivo de avaliálos frente às funcionalidades do artefato proposto. Nesse caso, serão identificados os possíveis requisitos impactados pela adoção de blockchain e, em seguida, pretende-se evidenciar, com base numa análise de dados empíricos secundários, as potencialidades e desafios associados. Adicionalmente, pretende-se conduzir um experimento computacional de modo que se possa investigar quantitativamente as seguintes métricas: i) consumo de memória da blockchain, ii) tempo de mineração das transações, iii) tempo de busca de dados e iv) número de transações por bloco e v) latências das requisições GET e POST. Tal experimento, de escopo quantitativo, faz-se relevante visto que o desempenho demonstra-se como um requisito de qualidade necessário nos sistemas de forma a impactar diretamente na experiência do usuário.

\section{Considerações Finais}

A partir do planejamento apresentado, este trabalho contribui ao compartilhar um desenho de pesquisa sociotécnico no contexto de uma solução baseada em blockchain para PEP. Esta tecnologia demonstra-se pertinente para o contexto de PEP devido a viabilização de partes completamente anônimas no compartilhamento de informações, de forma a garantir a confiabilidade, transparência e privacidade aos dados do paciente. Tais características permitem ao paciente o controle dos seus próprios dados clínicos. Dessa forma, observase a necessidade de um entendimento, a partir de um ponto de vista colaborativo, de como tais benefícios impactam diretamente os usuários de forma individual e coletiva. Com base neste enquadramento, planeja-se como trabalhos futuros a implementação da proposta e sua devida avaliação de modo que se avance em tal domínio a discussão de elementos sociais e técnicos em consonância aos requisitos da SBIS e LGPD. 


\section{Referências}

Agostinho, B., Schreiner, G., Gomes, F., Pinto, A. S. R., and Dantas, M. (2019). Unificação de dados de saúde através do uso de blockchain e smart contracts. In Anais da XV Escola Regional de Banco de Dados, pages 31-40. SBC.

Bassan de Moraes, C. R., Valentim, M. L., and Pinheiro de Souza, L. P. (2019). Recursos informacionais para a construção do conhecimento em empresas de software: Abordagem sistêmica. Brazilian Journal of Information Science, 13(3).

Beck, R., Avital, M., Rossi, M., and Thatcher, J. B. (2017). Blockchain technology in business and information systems research. Business Information Systems Engineering, 59:381-384.

Beck, R., Weber, S., and Gregory, R. W. (2013). Theory-generating design science research. Information Systems Frontiers, 15(4):637-651.

Bowen, G. A. et al. (2009). Document analysis as a qualitative research method. Qualitative research journal, 9(2):27.

CFM (2002). Resolução CFM No 1.638/2002, de 01 de novembro de 2018. Define prontuário médico e torna obrigatória a criação da Comissão de Revisão de Prontuários nas instituições de saúde. Brasília, DF, 9 de ago. 2002.

Costa, A. P., Loureiro, M. J., and Reis, L. P. (2014). Do modelo 3c de colaboração ao modelo 4c: Modelo de análise de processos de desenvolvimento de software educativo. Revista Lusófona de Educação, (27):181-200.

Cukierman, H. L., Teixeira, C., and Prikladnicki, R. (2007). Um olhar sociotécnico sobre a engenharia de software. Revista de Informática Teórica e Aplicada, 14(2):199-219.

da Conceição, A. F., da Silva, F. S. C., Rocha, V., Locoro, A., and Barguil, J. M. (2018). Eletronic health records using blockchain technology. arXiv preprint arXiv:1804.10078.

Davis, F. D. (1989). Perceived usefulness, perceived ease of use, and user acceptance of information technology. MIS quarterly, pages 319-340.

Massad, E., Marin, H. d. F., and Azevedo Neto, R. S. d. (2003). O prontuário eletrônico do paciente na assistência, informação e conhecimento médico. pages 1-202.

McFarlane, C., Beer, M., Brown, J., and Prendergast, N. (2017). Patientory: a healthcare peer-to-peer emr storage nntwork v1. Entrust Inc.: Addison, pages 1-19.

Morgan, D. L. (1996). Focus groups. Annual review of sociology, 22(1):129-152.

Nicolaci-da Costa, A. M. and Pimentel, M. (2011). Sistemas colaborativos para uma nova sociedade e um novo ser humano. Sistemas colaborativos. PIMENTEL, M.; FUKS, H.(Orgs.). Rio de Janeiro: Elsevier.

Nielsen, J. (1994). Usability engineering. Morgan Kaufmann.

Prinz, W. (2018). Blockchain and cscw-shall we care? In Proceedings of 16th European Conference on Computer-Supported Cooperative Work-Exploratory Papers. European Society for Socially Embedded Technologies (EUSSET).

Ricarte, I. L. (2019). Sistemas nacionais de prontuários eletrônicos frente à privacidade de dados. E-prints in library information science. Disponível em: http://eprints.rclis.org/33929/.

Rubin, J. and Chisnell, D. (2008). Handbook of usability testing: how to plan, design and conduct effective tests. John Wiley \& Sons.

Vaishnavi, V. and Kuechler, B. (2004). Design Research in Information Systems.

Viana, C., Brandão, A., Dias, D., and Castellano, G. (2020). Blockchain para gerenciamento de prontuários. RISTI-Revista Ibérica de Sistemas e Tecnologias de Informação, pages 177-187.

Zhang, P., White, J., Schmidt, D. C., Lenz, G., and Rosenbloom, S. T. (2018). Fhirchain: applying blockchain to securely and scalably share clinical data. Computational and Structural Biotechnology Journal, 16:267-278. 